\title{
Dementia and hearing loss: a narrative review
}

\author{
Manisha Ray ${ }^{1}$ \\ Tom Dening ${ }^{2 *}$ \\ Brian Crosbie ${ }^{3}$
}

${ }^{1}$ Nottinghamshire Healthcare NHS Foundation Trust, Nottingham, UK

${ }^{2}$ Division of Psychiatry \& Applied Psychology, School of Medicine, University of Nottingham, UK

${ }^{3}$ Faculty of Medicine \& Health Sciences, University of Nottingham, UK

*Institute of Mental Health, Triumph Road, Nottingham, NG7 2TU, UK

+441158230421

Tom.dening@nottingham.ac.uk 


\begin{abstract}
Dementia and hearing loss are highly prevalent in older people. Both these conditions together increase complexities in all aspects of an individual's care and management plan. There has been increasing research interest about the relationship between dementia and hearing loss in recent years. In this review we discuss the relationship between hearing loss and dementia, including hearing loss as a risk factor for dementia; the effects of dementia with hearing loss on affected persons' quality of life and the care they receive; screening and available interventions; and opportunities for prevention. We also discuss dementia and hearing loss in the care home setting, as the majority of residents have either, or indeed both, dementia and/or hearing loss. Several mechanisms have been suggested for how hearing loss and dementia may be related but the evidence for how these may operate together is still unclear. Similarly, although it is to be hoped that the active identification and management of hearing problems may help to reduce the future development of cognitive impairment, evidence for this is still lacking.
\end{abstract}

\title{
Highlights
}

- Hearing loss and dementia are often associated - they may occur together, though longitudinal studies suggest that hearing loss may be a genuine risk factor for subsequent dementia.

- There may be several mechanisms that contribute to the association, including brain pathology but also social factors. The relationship of central hearing loss with dementia needs further exploration.

- Multiple barriers exist in both screening and treatment of hearing loss among people with dementia emphasising the need for an integrated patient centred approach.

- Hearing loss is linked to multiple physical and psychological comorbidities in persons with dementia.

- Extended clinical trials are needed to establish whether treating hearing loss is effective in the prevention of dementia. 


\section{Dementia and hearing loss: a narrative review}

\section{Introduction}

Dementia and hearing loss are both common conditions in the ageing population and they often coexist [1]. Hearing loss in the context of dementia is an important current topic; for example hearing loss as a risk factor for the development of dementia, and the challenges of assessing and managing hearing loss in people who have cognitive impairment. This narrative review discusses the relationship between hearing loss and dementia, including hearing loss as a risk factor for dementia; the effects of dementia with hearing loss on affected persons' quality of life and the care they receive; screening and available interventions; and opportunities for prevention. We also discuss dementia and hearing loss in the care home setting, as the majority of residents have either, or indeed both, dementia and/or hearing loss.

For the review, we undertook a comprehensive search, including Medline, Psychinfo, Embase, Cinahl, BNI databases, and Google Scholar. The main searches were: dementia and hearing loss; cognitive impairment and hearing loss and/or deafness. These yielded over 1500 titles, though with many duplicates. After discarding papers that were clearly not relevant, 272 titles were obtained from the main search and abstracts reviewed for all of these, selecting papers of high interest for full-text review and possible inclusion in the paper. Subsequently, we also did smaller more focused searches for specific topics such as prevention and central hearing loss. We did not use formal inclusion or exclusion criteria: a paper was included if it appeared relevant and important to the topic, and papers were excluded if they did not meet this threshold or if they duplicated findings available in other, stronger papers.

To limit the number of references that could be included for the review, we have focused on papers that are recent and influential, as judged by their likely impact. This was done by consensus discussion between MR and TD. Papers were selected from journals with higher impact factors, or if they were frequently cited by other researchers, or if they presented novel and interesting findings. Papers were from the last ten years, except for some topics where no recent research had been reported, in which case an older paper was included. Decisions on inclusion were a matter of authorial judgement rather than any formal process.

\section{Relationship between dementia and hearing loss}

Hearing loss and dementia both are highly prevalent in older population. For example, the prevalence of hearing loss in the UK for $71-80$ years olds is around $42 \%$ for men and $39 \%$ for women [2] but increases sharply beyond the age of 80 . The prevalence of dementia in over 
65s in the UK is around 6.5\% [3] but again the prevalence increases steeply with age, so the prevalence at age $85-90$ is around $17 \%$ and for $>90$ it is about $30 \%$. Among certain groups of the population, notably care home residents, both conditions are so common as to affect the majority $[4,5]$.

Currently, there are around 50 million people living with dementia worldwide and this number is likely to grow by at least three times (to 152 million) by 2050 [6]. As well as this, over $5 \%$ of world's population (466 million people) suffer from disabling hearing loss, including about one-third of the global over-65 population. This number is predicted to rise to over 900 million by 2050 [7]. Both of these conditions have huge economic impact. WHO estimates that unaddressed hearing loss poses an annual global cost of US\$ 750 billion [7] and the current annual cost of dementia is about US\$ 1 trillion [6], predicted to double by 2030.

As both of these conditions are age-related and have high prevalence in older age groups, hearing loss and dementia often co-exist. For example, Nirmalasari et al (2017) found that $60 \%$ of people in a tertiary memory clinic had at least a mild hearing loss even in their better hearing ear [8].

One of the earliest papers to report an association between hearing loss and dementia was a case control study [9], which found that cases of Alzheimer's disease had twice the prevalence of $30 \mathrm{~dB}$ hearing loss compared to controls (odds ratio $=2.0,95 \%$ confidence interval, 1.2 to 3.4). Since then, numerous papers, including large longitudinal studies, systematic reviews and meta-analysis have reported similar findings [10-16]. There appears to be a dose dependent relationship, with greater hearing loss being related to more severe dementia [10] (mild hearing loss: hazard ratio for incident dementia $=1.89$ (95\% Cl, 1.00 3.58); moderate hearing loss: $\mathrm{HR}=3.00(95 \% \mathrm{Cl} 1.43-6.30)$; severe hearing loss: $\mathrm{HR}=4.94$ (95\% $\mathrm{Cl} 1.09-22.40)$. It has also been suggested laterality of hearing loss may be important, with one study finding a greater likelihood of cognitive impairment with hearing loss in the right ear [17].

However, a cross-sectional association does not provide any information about how the two conditions are related, how they progress over time, and what mechanisms may underlie them. Therefore there has been increasing interest in longitudinal studies to investigate these questions. These provide evidence that hearing loss is linearly associated with dementia and, the more severe the baseline hearing impairment, the stronger is the association between hearing loss and incident dementia. In the Baltimore Longitudinal Study of Aging, the risk of incident all-cause dementia increased almost twice, three and five times in individuals with mild, moderate and severe hearing loss respectively. For every 10 $\mathrm{dB}$ hearing loss, the risk of incident Alzheimer's disease increased by 1.2 times [11]. 


\section{Does hearing loss cause dementia?}

The mechanism underlying the association between hearing loss and dementia is not yet clear [18] though several possible mechanisms have been suggested [19]. These are as follows:

\section{Increased cognitive load}

Hearing loss is likely to increase the cognitive effort required to process speech, since reduced or distorted sensory input will require the brain to work harder to make sense of it [20]. It is then hypothesised that excessive cognitive load leads to changes in brain structure and further neurodegeneration, though direct evidence for this is limited.

\section{Changes in brain structure and function}

The Baltimore Longitudinal Study of Aging [21] reported that hearing impairment was linked to accelerated brain atrophy of whole brain and regional volume reduction of the right temporal lobe. Chronic hearing loss leads to reduced activation of central auditory pathway, dysfunction of auditory-limbic pathway and deafferentation-induced atrophy of frontal lobe [22]. Hearing loss has also been linked to atrophy of the hippocampus [23].

\section{Common cause}

This hypothesis suggests a common mechanism causing both hearing loss and cognitive decline. The two conditions arise from the same cause but do not interact or cause each other. There are several common risk factors for both hearing loss and cognitive decline, mainly vascular risk factors, such as smoking and diabetes that increase the risk of cardiovascular disease and stroke. It has been suggested that the APOE $\varepsilon 4$ allele may confer an increased risk but this is not established. The specific molecular link between hearing loss and dementia remains unknown [24].

\section{Social disengagement}

Individuals with hearing loss often find social interactions less satisfactory as it is difficult to filter out a conversation from background noise. As a result, they may tend to withdraw from social occasions, which leads to reduced social and cognitive stimulation, isolation and possibly also depression. Hearing loss is associated with increased odds $(\mathrm{OR}=3.5,95 \% \mathrm{Cl} 1.9$ 6.4) of social isolation in older people [25]. No longer using social skills or keeping up to date with social events causes loss of these abilities. It is likely that this process also has neural correlates, for example deterioration of auditory signal processing.

\section{Overdiagnosis of cognitive impairment}

Performance on the cognitive tests that are used in the assessment of possible dementia is affected by various factors, including sensory impairment $[26,27]$. If a person is not able to hear the question that is being asked of them, they are obviously less likely to supply a 
correct answer. This is a particular problem when assessments are performed in noisy environments, such as hospitals, though it is easy under any circumstances to underestimate the extent to which hearing loss is contributing to impaired test performance. Thus, it would be possible to misdiagnosis a person with normal cognition as having dementia, which has obvious adverse consequences for them.

\section{Hearing loss as a prodromal feature (harbinger) of dementia}

Central auditory processing refers to the brain's ability to make sense out of the sounds received by the cochlea. It is therefore vulnerable to neurodegeneration and there is evidence that central auditory processing may be affected early in the course of Alzheimer's disease and in mild cognitive impairment [28]. A person may perform normally on such tests as pure tone audiometry but with impaired speech perception. In this way, symptoms that are due to brain disease may present as and be treated as hearing loss.

Each of these postulated mechanisms is plausible, so it is a challenge for future research to test the extent to which each may operate.

\section{Interventions for hearing loss in dementia}

Hearing loss is often not detected and/or not treated in people with dementia. NICE guidance on hearing loss [29] recommends that a hearing assessment by an audiologist should be considered for every person with possible dementia or mild cognitive impairment, and should be considered every two years in people with a diagnosis of dementia if they have not been previously diagnosed with hearing loss. The barriers to screening or treatment of hearing loss may include denial of the problem, reluctance to undergo testing and poor compliance with hearing aids.

Nonetheless, treating hearing loss in people with dementia is of potential benefit both to the person with dementia and to their families and carers. It may help to improve communication, and thereby increase quality of life and reduce behavioural symptoms of dementia. This in turn may reduce stress for carers. However, the quality of the evidence is limited, most of it consisting of quasi-experimental studies and case studies [30]. The only randomised controlled trial reported no significant improvement in cognitive [31] or behavioural outcomes [32]. To date, therefore, there is no consistent evidence that hearing interventions are of benefit for cognitive function, cognitive decline, quality of life or caregiver burden, and there is scope for future research [33].

Hearing aids are most commonly used intervention for hearing loss among people with dementia. Nirmalasari et al [8] found a high prevalence (60\%) of hearing loss in a memory clinic cohort but also low utilization of hearing aids among people with cognitive impairment, with only $54 \%$ of individuals with moderate or severe hearing loss using aids. 
Lin and Albert [34] commented that hearing aids were more likely to be used by people in better health and of higher socioeconomic status, and recommended that clinicians should take a more active role in seeking hearing assessments and offering treatment.

Other interventions, such as hearing amplification devices and focused communication strategies, have been applied in both in-patient [35] and out-patient settings [36] with some evidence of benefit on outcomes such as communication and depression. There is so far little research on the use of cochlear implantation in treatment of severe to profound hearing loss among people with cognitive impairment or dementia. Mosnier et al [37] reported improvements in cognitive function after cochlear implantation in some patients who had mild cognitive impairment $(\mathrm{MCl}$ ) at baseline (including $10 / 31(32 \%)$ returning to the normal range of cognitive function).

In terms of improving current practice, what appears to be required is increased awareness of hearing loss and its impact among professionals working with dementia, and increased awareness of cognitive impairment and dementia among speech-language therapists and audiologists. There is to date relatively little literature about interdisciplinary working though there are encouraging findings from educational initiatives [38] and service developments, for instance dementia audiology services in acute hospitals. Management of hearing loss and communication should be integrated into the person's broader dementia care [36] and delivered as close to the person's own setting as possible. Involving speech and language therapists working with audiologists can greatly increase opportunities for meaningful engagement in persons with dementia [39].

\section{Hearing loss and other symptoms of dementia}

Age-related hearing loss has been independently linked not just with dementia but also other conditions such as depression and falls $[40,41]$. Hearing loss and dementia both have a strong link with frailty [21], and indeed sensory impairment is a feature of some of the frailty assessment scales that are in use.

Dementia and hearing loss are both independent risk factors for delirium[42] and their effects are likely to interact, especially in noisy environments like acute hospitals. Hearing loss increases the likelihood of all forms of psychotic symptoms [43] and hallucinations in dementia also occur more commonly in people with sensory impairments, both visual and auditory [44].

Both hearing loss and dementia may cause social withdrawal and impairments in communication, functioning, participation in activities [39]. Among nursing home residents sensory impairments has been associated with low social engagement and low time spent in activities [45]. These features may in turn be linked to low mood and depression, and the evidence suggests a link between lack of social involvement and behavioural symptoms of 
dementia such as agitation and aggression $[46,47]$. A range of behavioural and psychological symptoms of dementia, including depression, agitation and aggression, can be exacerbated by untreated hearing loss in people with dementia [48].

Hearing interventions for people with dementia have been reviewed by Dawes et al [34]. They identified ten studies, mainly studying the effects of hearing aid provision. Most were case reports or case series, and outcomes included behavioural symptoms, quality of life and caregiver burden. Case series and reports were more likely to show improvements in behaviour, e.g. Haque et al [49], but the only randomised controlled trial [32] reported no evidence of benefit in any outcome measures.

The assessment and management of comorbid physical or psychological problems in people with dementia therefore need to consider the possible coexistence of hearing loss and how it may be exacerbating the impact of such conditions.

\section{Hearing loss and dementia in care home residents}

Hearing loss and dementia are both common in residents in long term care settings. Furthermore, dementia with severe hearing impairment increases the risk of institutionalisation among older adults aged 75 or older [50]. Among care home residents, the majority have hearing loss, with prevalence rates up to $90 \%$ reported [51]. The prevalence of dementia in UK care residents is around 75\% [4], so a large proportion of residents will have both conditions, not to mention other long-term conditions that contribute to frailty.

As both conditions affect communication, challenges in communication will frequently arise for the staff working in long term care facilities. In turn, this has potential negative effects on the provision of care and quality of life of the residents. Staff members often find it hard to differentiate the relative contributions of hearing loss and dementia when communication breaks down. However, knowing the individual helps them to distinguish between these two factors [52].

Despite the high prevalence of hearing loss, hearing aids appear to be underused [53]. There are numerous reasons for this, starting with failure to identify hearing loss in many residents. It may, for example, be assumed that a person's failure to understand may arise from their dementia and not from inability to hear what is being said. Assessing the degree of hearing loss in residents with dementia may be difficult, as too may be the fitting of hearing aids. Once supplied, hearing aids may pose difficulties in their use; for example, if they fit poorly, are not set at the right volume, or have flat batteries, and they are liable to get lost or broken [54]. Most residents need help with using hearing aids but staff often lack confidence in managing them or simply do not think the task is of value. It has also been suggested that cost may be a factor in poor uptake of hearing aids [55] though this is less 
likely in a publicly-funded health service such as the NHS. Cost is significant in that privately purchased hearing aids are likely to be smaller and more comfortable but, conversely, more easily mislaid.

Staff attitudes and their level of training are clearly important. For example, staff have often not received training in the use or maintenance of hearing aids, or they may rely on relatives to deal with them [56]. Other issues that may act as barriers to managing hearing loss in care home settings are impaired communication between care staff and external professionals; lack of staff involvement due to issues such as lack of appropriate emphasis in policies on the importance of hearing loss; lack of suitable education and training; and low priority of management of hearing loss over other demands [57].

There is something of a paradox in that there is relatively little research regarding good practice or effective interventions to improve hearing-related communication in care home residents with dementia yet, because these conditions are so common, there must be immense practical day-to-day experience among staff. This state of affairs lends itself well to realist enquiry and questions of what works, for whom and under what circumstances? A recent realist review [58] developed a programme theory with five components: 1) positive regard and empathy for residents - leadership promotion of person centred care; 2) training on hearing loss and dementia to raise importance of hearing-related communication; 3 ) knowing the person and awareness; 4) supporting and monitoring of residents' hearingrelated communication needs; 5 ) managing noise in the care home environment. These components were all linked together by the concept of 'permission', which reflects how staff can feel that they have approval to seek meaningful interaction with residents, and to see this as an important part of their work. Leadership and management can intervene in different ways to indicate to staff that they are permitted to practice with a level of autonomy that best serves the hearing-related communication needs of residents.

\section{Hearing loss and prevention of dementia}

Hearing loss has been identified as an important potentially modifiable risk factor for dementia, with a population attributable risk fraction (PAF) of $9.1 \%$ (relative risk $=1.9,95 \%$ $\mathrm{Cl}$ 1.4-2.7) [18]. However, it remains unclear if prevention of hearing loss can delay the onset or reduce the incidence of dementia. It is still of course important to bear in mind that hearing aids have the potential to improve functioning, quality of life and delay the progress of dementia [30].

The available interventional studies of hearing interventions to prevent dementia, with cognition as the main outcome measure, show mixed findings [59]. To date, three nonrandomised controlled trials $[60,61,62]$ reported improvement in cognitive outcome following treatment of hearing loss with hearing aids. Conversely, three non-randomised controlled trials $[63,64,65]$ failed to find any evidence of benefit. The evidence is therefore 
conflicting and existing studies are of low to moderate quality as they are liable to various biases, e.g. hearing aid users may be more affluent or otherwise healthier than non-users.

Findings from studies of cochlear implantation as treatment for severe or profound hearing loss in people aged 65 and older have shown improvements in various outcomes, including cognitive function, mood and quality of life, following the implantation $[37,66,67]$ but whether this procedure is effective in protecting against subsequent dementia is not clear. Cosetti et al [68] followed their patients for a mean of 3.7 years but the numbers were small $(\mathrm{N}=7)$ and in the absence of a control group it is difficult to interpret the findings.

There are several challenges that exist in designing well controlled hearing intervention studies with long term cognitive outcome [59]. These include the need for an adequate sample size and duration of follow-up; the expense of such studies; practice effects in cognitive measures in a longitudinal study; non-adherence to hearing aids or other causes of attrition from the trial; and ethical considerations in intervention studies, notably withholding recognised and available treatments from the control group. Despite these challenges, and following a successful feasibility trial [69], the ACHIEVE trial is currently underway, aiming to recruit 850 participants, with the primary outcome being cognitive function at 3 years follow-up [70]. Obviously, the results of this trial will be eagerly awaited.

\section{Central hearing loss}

Central hearing loss (also known as central auditory processing disorder or central presbycusis), as opposed to sensorineural or conductive types of hearing loss, is characterised by difficulty in making sense of speech against noise [71]. In a conventional hearing test, the affected person may report not being able to hear but, in fact, pure tone audiometry is relatively normal while tests like speech recognition thresholds or word recognition scores are impaired. An affected person is likely to consider themselves deaf, even though they appear to respond to sounds in their environment. Thus, central hearing loss may be easily overlooked on testing or it may be regarded as 'functional' because of the variable and apparently inconsistent presentation. It may arise from a variety of brainstem and cortical lesions involving auditory pathways. Its prevalence is related to increasing age, for example $14.3 \%$ in a sample of nearly 500 people of mean age 72.8 years (SD 6.2) [72]. Of course it may coexist with more peripheral causes of hearing loss, which may also make its presence harder to detect.

Central hearing loss appears to be commoner in mild cognitive impairment and Alzheimer's disease than in age-matched controls (odds ratios 1.6 and 4.1 respectively; [72]. It may therefore be that deficits of central auditory processing are potential biomarkers for neurodegeneration [73], though at the moment there is little research that has linked hearing loss with the conventional biomarkers for Alzheimer's disease [74]. 
The importance of central hearing loss therefore merits further attention in disentangling the relationship between hearing loss and cognitive impairment. Managing it requires other approaches than hearing aids and so it may be a less amenable risk factor than other types of hearing loss in attempts to prevent the development of dementia [18].

\section{Discussion}

In summary, hearing loss and dementia are common age-related problems and frequently occur together, and in association with other conditions. It appears that older people with hearing loss are at increased risk of developing cognitive impairment and dementia; however, the mechanisms for this are unresolved. There are equally plausible biological and psychosocial reasons. There remains an urgent need for a well-designed trial to test whether hearing interventions can help to prevent dementia. In the meantime, other research should concentrate on implementing what we already know of good practice, for example developing specialist expertise among audiologists and speech and language therapists in relation to dementia, and improving access for older people to such services.

A final question is whether hearing impairment has distinct features that warrant separate study or whether it just forms a component of sensory deprivation alongside other impairments such as visual loss. There is a tendency in recent research to look at sensory impairments together and this may be justified in that they may act together to pull affected people down towards a threshold where their independent functioning is compromised. However, hearing as a sensory modality has distinct features, in particular its intimate relationship with language and speech and, probably for this reason alone, research into hearing loss per se remains important.

\section{Declaration}

This paper presents independent research funded by the National Institute for Health Research (NIHR) under its Research for Patient Benefit (RfPB) Programme (Grant Reference Number PB-PG-0815-20013). The views expressed are those of the author(s) and not necessarily those of the NIHR or the Department of Health and Social Care.

\section{Contributions of authors}

Manisha Ray performed literature searches, selection of papers for inclusion, and wrote the paper. Tom Dening conceived of the review, selected papers for inclusion and wrote the review. Brian Crosbie advised about the content of the review and wrote the paper.

The authors have no declarations of interest. 


\section{References}

1. World Health Organization. Risk Reduction of Cognitive Decline and Dementia. 2019. https://www.who.int/mental_health/neurology/dementia/guidelines_risk_reduction/e n/ (accessed 29/05/19).

2. MA. Akeroyd, K. Foreman, JA. Holman. Estimates of the number of adults in England, Wales, and Scotland with a hearing loss. Int J Audiol. 53(1) (2014) 60-61. doi: 10.3109/14992027.2013.850539.

3. FE. Matthews, A. Arthur, LE. Barnes, J. Bond, C. Jagger, L. Robinson, et al. A two-decade comparison of prevalence of dementia in individuals aged 65 years and older from three geographical areas of England: results of the Cognitive Function and Ageing Study I and II. Lancet. 382(9902) (2013): 1405-1412. doi: 10.1016/S0140-6736(13)61570-6.

4. AL. Gordon, M. Franklin, L. Bradshaw, P. Logan, R. Elliott, JRF. Gladman. Health status of UK care home residents: a cohort study. Age Ageing 43(1) (2014) 97-103. https://doi.org/10.1093/ageing/aft077.

5. M. Echalier. A World of Silence: the case for tackling hearing loss in care homes. London: Action on Hearing Loss. 2012.

6. Alzheimer's Disease International. World Alzheimer's Report 2018. https://www.alz.co.uk/research/world-report-2018. (accessed 29/05/19).

7. World Health Organization. Deafness Prevention. https://www.who.int/deafness/en/. (accessed 29/05/19).

8. O. Nirmalasari, SK. Mamo, CL. Nieman, A. Simpson, J. Zimmerman, MA. Nowrangi, FR. Lin, ES. Oh. Age related hearing loss in older adults with cognitive impairment. International Psychogeriatrics 29(1) (2017) 115-121. doi: 10.1017/S1041610216001459

9. RF. Uhlmann, EB Larson, TS Rees, TD Koepsell, LG Duckert. Relationship of hearing impairment to dementia and cognitive dysfunction in older adults. JAMA. 261(13) (1989) 1916-1919.

10. FR. Lin, E. J. Metter, RJ. O’Brien, SM. Resnick, AB. Zonderman, L. Ferrucci. Hearing loss and incident dementia. Arch Neurol. 68(2) (2011). 214-220.

doi: 10.1001/archneurol.2010.362

11. J. Gallacher, V. Ilubaera , Y. Ben-Shlomo, A. Bayer, M. Fish, W. Babisch, P Elwood. Auditory threshold, phonologic demand, and incident dementia. Neurology. 79(15) (2012) 1583-1590. doi: 10.1212/WNL.0b013e31826e263d.

12. JA. Deal , J. Betz, K. Yaffe , T. Harris, E. Purchase-Helzner, S. Satterfield et al. Hearing impairment and incident dementia and cognitive decline in older adults: the HealthABC study, J Gerontol A Biol Sci Med Sci. 72(5) (2017) 703-709. doi: 10.1093/gerona/glw069.

13. M. Osler, GT. Christensen, EL. Mortensen, K. Christensen, E. Garde, MP. Rozing. Hearing loss, cognitive ability, and dementia in men age $19-78$ years. Eur J Epidemiol. 34(2)(2019) 125-130. doi: 10.1007/s10654-018-0452-2. 
14. AH. Ford, GJ. Hankey, BB. Yeap, J. Golledge, L. Flic.ker, OP. Almeida. Hearing loss and the risk of dementia in later life. Maturitas, 112 (2018) 1-11. doi: 10.1016/j.maturitas.2018.03.004.

15. J. Wei, Y. Hu, L. Zhang, Q. Hao, R. Yang, H. Lu, X. Zhang, EK. Chandrasekar. Hearing impairment, mild cognitive impairment, and dementia: a meta-analysis of cohort studies. Dement Geriatr Cogn Disord Extra. 7 (2017) 440-452 DOI: 10.1159/000485178.

16. RS. Thomson, P. Auduong, AT. Miller, RK. Gurgel. Hearing loss as a risk factor for dementia: a systematic review. Laryngoscope Investig Otolaryngol. 2(2) (2017) 69-79. doi: $10.1002 /$ lio2.65.

17. MJ. Huh. The relationships between cognitive function and hearing loss among the elderly. Journal of Physical Therapy Science. 30(1) (2018) 174-176 doi: 10.1589/jpts.30.174.

18. G. Livingston, A. Sommerlad, V. Orgeta, SG. Costafreda, J. Huntley, D. Ames, et al. Dementia prevention, intervention, and care. Lancet. 390 (2017) 2673-2734. doi: https://doi.org/10.1016/S0140-6736(17)31363-6.

19. Y. Uchida, S. Sugiura, Y. Nishita, N. Saji , M. Sone, H. Ueda. Age related hearing loss and cognitive decline- The potential mechanism linking the two, Auris Nasus Larynx. 46(1) (2019) 1-9. doi: 10.1016/j.anl.2018.08.010.

20. F. Panza, V. Solfrizzi, D. Seripa, BP. Imbimbo, R. Capozzo, N. Quaranta et al. Age-related hearing impairment and frailty in Alzheimer's disease: interconnected associations and mechanisms. Front Aging Neurosci. 7 (2015) 113. doi: 10.3389/fnagi.2015.00113.

21. FR. Lin , L. Ferrucci, Y. An, JO. Goh, J. Doshi, EJ. Metter et al. Association of hearing impairment with brain volume changes in older adults, Neuroimage. 90 (2014) 84-92. doi: 10.1016/j.neuroimage.2013.12.059.

22. BR. Rutherford, K. Brewster, JS. Golub, AH. Kim, SP. Roose. Sensation and psychiatry: linking age related hearing loss to late life depression and cognitive decline. Am J Psychiatry. 175 (3) (2018) 215-224. doi: 10.1176/appi.ajp.2017.17040423.

23. Y. Uchida, Y. Nishita, T. Kato, K. Iwata, S. Sugiura, H. Suzuki et al. Smaller hippocampal volume and degraded peripheral hearing among Japanese community dwellers. Front Aging Neurosci. 10 (2018) 319. doi: 10.3389/fnagi.2018.00319. eCollection 2018.

24. U. Shen, B. Ye, P. Chen, Q. Wang, C. Fan, Y. Shu, M. Xiang. Cognitive decline, dementia, Alzheimer's disease and presbycusis: examination of the possible molecular mechanism. Front Neurosci 12 (2018) 394. doi: 10.3389/fnins.2018.00394.

25. P. Mick, I. Kawachi, FR. Lin. The association between hearing loss and social isolation in older adults. Otolaryngol Head Nech Surg. 150(3)(2014) 378-384. doi: 10.1177/0194599813518021.

26. K. Dupuis, MK. Pichora-Fuller, AL. Chasteen, V. Marchuk, G. Singh, SL. Smith. Effects of hearing and vision impairments on the Montreal Cognitive Assessment. Neuropsychol Dev Cogn B Aging Neuropsychol Cogn 22(4) (2015) 413437.doi:10.1080/13825585.2014.968084. 
27. LE. Jorgensen, CV. Palmer, S. Pratt, KI. Erickson, D. Moncrieff, The effect of decreased audibility on MMSE performance: a measure commonly used for diagnosing dementia. J Am Acad Audiol. 24(4) (2016) 311-323. doi: 10.3766/jaaa.15006.

28. E. Idrizbegovic, C. Hederstierna, M. Dahlquist, C. Kämpfe Nordström, V. Jelic, U. Rosenhall. Central auditory function in early Alzheimer's disease and in mild cognitive impairment. Age Ageing. 40(2) (2011) 249-254. doi: 10.1093/ageing/afq168.

29. NICE. Hearing Loss in Adults: assessment and management. 2018. https://www.nice.org.uk/guidance/ng98. (accessed 29/05/19).

30. SK. Mamo, NS. Reed, C. Price, D. Occhipinti, A. Pletnikova, FR. Lin et al. Hearing loss treatment in older adults with cognitive impairment: a systematic review. J Speech Lang Hear Res. 61(10) (2018) 2589-2603. doi: 10.1044/2018_JSLHR-H-18-0077.

31. MF. Nguyen, M. Bonnefoy, A. Adrait, M. Gueugnon, C. Petitot, L. Collet et al. Efficacy of hearing aids on the cognitive status of patients with Alzheimer's disease and hearing loss: a multicenter controlled randomized trial. J Alzheimers Dis. 58(1) (2017) 123-137. doi: 10.3233/JAD-160793.

32. A. Adrait, X. Perrot, MF. Nguyen, M. Gueugnon ' C. Petitot, L. Collet et al. Do hearing aids influence behavioral and psychological symptoms of dementia and quality of life in hearing impaired Alzheimer's disease patients and their caregivers?. J Alzheimers Dis. 58 (01) (2017) 109-121. doi: 10.3233/JAD-160792.

33. P. Dawes, L Wolski , I. Himmelsbach, J. Regan, I. Leroi. Interventions for hearing and vision impairment to improve outcomes for people with dementia: a scoping review. Int Psychogeriatr. 31(2) (2019) 203-221. doi: 10.1017/S1041610218000728.

34. FR. Lin, M. Albert. Hearing loss and dementia: who is listening? Aging Ment Health. 18(6) (2014) 671-673. doi: 10.1080/13607863.2014.915924

35. A Shukla, CL. Nieman, C. Price, M. Harper, FR. Lin, NS. Reed. Impact of hearing loss on patient-provider communication among hospitalized patients: a systematic review. Am J Med Qual. 34(3)( 2019) 284-292.doi: 10.1177/1062860618798926

36. SK. Mamo, O. Nirmalasari , Cl. Nieman, MK. McNabney, A. Simpson, ES. Oh et al. Hearing Care Intervention for Persons with Dementia: A Pilot Study. Am J Geriatr Psychiatry. 25(1) (2017) 91-101. doi: 10.1016/j.jagp.2016.08.019.

37. I. Mosnier, A. Vanier, D. Bonnard, G. Lina-Granade, E. Truy, P. Bordure et al. Long-term cognitive prognosis of profoundly deaf older adults after hearing rehabilitation using cochlear implants. J Am Geriatr Soc. 66(8) (2018) 1553-1561. doi: 10.1111/jgs.15445.

38. WA. Kaf, LS. Barboa, BJ. Fisher, LA. Snavely. Effects of interdisciplinary service learning experience for audiology and speech-language pathology students working with adults with dementia. American Journal of Audiology. 20 (2011).S241-S249. doi.org/10.1044/1059-0889(2011/10-0025).

39. HI. Hubbard, SK. Mamo, T. Hopper. Dementia and hearing loss: interrelationships and treatment considerations. Semin Speech Lang. 39(3) (2018) 197-210. doi: 10.1055/s0038-1660779. 
40. JA. Deal, NS. Reed, AD. Kravetz, H. Weinreich, C. Yeh, FR. Lin, et al. Incident hearing loss and comorbidity: a longitudinal administrative claims study. JAMA Otolaryngol Head Neck Surg. 145(1) (2019) 36-43. doi:10.1001/jamaoto.2018.2876.

41. E. Schonfeld , LT. Meyer, A. Becker, K. Tate, M. Moodabagil ,C. McSharry et al. Correlation of hearing loss and chronic falling among patients with dementia in 3 memory-care communities. Annals of Long-Term Care. 26(2) (2018) 21-25. doi: 10.25270/altc.2018.04.00022.

42. TG. Fong, D. Davis, ME. Growdon, A. Albuquerque, SK. Inouye. The Interface of Delirium and Dementia in Older Persons. Lancet Neurol. 14(8) (2015) 823832. doi: 10.1016/S1474-4422(15)00101-5.

43. MM. Linszen, RM. Brouwer, SM. Heringa, IE. Sommer, Increased risk of psychosis in patients with hearing impairment: Review and meta-analyses. Neurosci Biobehav Rev.62(2016)1-20. doi: 10.1016/j.neubiorev.2015.12.012.

44. M. El Haj, J. Roche, R. Jardri, D. Kapogiannis, K. Gallouj, P. Antoine. Clinical and neurocognitive aspects of hallucinations in Alzheimer's disease. Neuroscience \& Biobehavioral Reviews. 83 (2017) 713-720. doi: 10.1016/j.neubiorev.2017.02.021.

45. HE. Resnick, BE. Fries, LM. Verbrugge. Windows to their world: the effect of sensory impairments on social engagement and activity time in nursing home residents. J Gerontol B Psychol Sci Soc Sci. 52(3) (1997) S135-44. doi:10.1093/geronb/52b.3.s135.

46. J. Cohen-Mansfield, K. Thein, MS. Marx, M. Dakheel-Ali, H. Murad, LS. Freedman. The relationships of environment and personal characteristics to agitated behaviors in nursing home residents with dementia. J Clin Psychiatry. 73(3) (2012) 392-399. doi: 10.4088/JCP.10m06605.

47. D. Dettmore, A. Kolanowski, M. Boustani. Aggression in persons with dementia: use of nursing theory to guide clinical practice. Geriatr Nurs. 30(1) (2009) 8-17. doi: 10.1016/j.gerinurse.2008.03.001.

48. SK. Mamo, E. Oh, FR. Lin. Enhancing communication in adults with dementia and agerelated hearing loss. Semin Hear. 38(2) (2017) 177-183. doi: 10.1055/s-0037-1601573.

49. R. Haque, N. Abdelrehman, Z. Alavi. 'There's a monster under my bed': hearing aids and dementia in long-term care settings. Annals of Long-Term Care 20 (2012) 28-33.

50. A. Hajek, C. Brettschneider, C. Lange, T. Posselt, B. Wiese, S. Steinmann et al. Longitudinal predictors of institutionalization in old age. PLoS One. 10(12) (2015) e0144203. doi: 10.1371/journal.pone.0144203. eCollection 2015.

51. T. Jupiter. Cognition and screening for hearing loss in nursing home residents. J Am Med Dir Assoc. 13(8) 2012 744-747. doi: 10.1016/j.jamda.2012.07.010.

52. SE. Slaughter, T. Hopper, C. Ickert, DF. Erin. Identification of hearing loss among residents with dementia: perceptions of health care aides. Geriatr Nurs. 35(6) (2014) 434-440. doi: 10.1016/j.gerinurse.2014.07.001.

53. J. Cohen-Mansfield, JW. Taylor. Hearing aid use in nursing homes. Part 1: prevalence rates of hearing impairment and hearing aid use. J Am Med Dir Assoc. 5(5) (2004) 283288. doi: https://doi.org/10.1016/S1525-8610(04)70017-1. 
54. T. Hopper, P. Hinton. Hearing loss among individuals with dementia: barriers and facilitators to care. Canadian Journal of Speech-Language Pathology and Audiology. 36(4) (2012) 302-313. Unique identifier: 2013-20842-003.

55. J. Cohen-Mansfield, DL. Infeld. Hearing aids for nursing home residents: current policy and future needs. Health Policy. 79(1) (2006) 49-56. doi: 10.1016/j.healthpol.2005.11.016.

56. J. Cohen-Mansfield, JW. Taylor. Hearing aid use in nursing homes. Part 2: barriers to effective utilization of hearing aids. J Am Med Dir Assoc. 5(5) (2004) 289-296. doi: https://doi.org/10.1016/S1525-8610(04)70018-3.

57. W. Wittich, F. Höbler, J. Jarry, KS. McGilton. Recommendations for successful sensory screening in older adults with dementia in long-term care: a qualitative environmental scan of Canadian specialists. BMJ Open 8 (2018) e019451. doi:10.1136/ bmjopen-2017019451.

58. B. Crosbie, M. Ferguson, G. Wong, D. Walker, S. Vanhegan, T. Dening. Giving permission to care for people with dementia in residential homes: learning from a realist synthesis of hearing-related communication. BMC Medicine. 17 (2019) 54. https://doi.org/10.1186/s12916-019-1286-9.

59. P. Dawes. Hearing interventions to prevent dementia. HNO. 67(3) (2019) 165-171. doi: 10.1007/s00106-019-0617-7.

60. A. Maharani, P. Dawes, J. Nazroo, G. Tampubolon, N. Pendleton; SENSE-Cog WP1 group. Longitudinal relationship between hearing aid use and cognitive function in older Americans. J Am Geriatr Soc. 66(6) (2018) 1130-1136. doi: 10.1111/jgs.15363.

61. H. Amieva, C. Ouvrard, C. Giulioli, C. Meillon, L. Rullier, JF. Dartigues. Selfreported hearing loss, hearing aids, and cognitive decline in elderly adults: a 25-year study. J Am Geriatr Soc. 63(10) (2015) 2099-104. doi: 10.1111/jgs.13649.

62. JA. Deal, AR. Sharrett, MS. Albert, J. Coresh, TH. Mosley, D. Knopman et al. Hearing impairment and cognitive decline: a pilot study conducted within the atherosclerosis risk in communities neurocognitive study. Am J Epidemiol. 181 (9) (2015) 680-690. doi: 10.1093/aje/kwu333.

63. P. Dawes, KJ. Cruickshanks, ME. Fischer, BE. Klein, R. Klein, DM. Nondahl. Hearingaid use and long-term health outcomes: Hearing handicap, mental health, social engagement, cognitive function, physical health, and mortality. Int J Audiol. 54(11) (2015) 838-844. doi: 10.3109/14992027.2015.1059503.

64. FR. Lin, K. Yaffe, J. Xia, QL. Xue, TB. Harris, E. Purchase-Helzner et al. Hearing loss and cognitive decline in older adults. JAMA Intern Med. 173(4) (2013) 293-299. doi: 10.1001/jamainternmed.2013.1868.

65. S.A. Valentijn, M.P. van Boxtel, S.A. van Hooren, H. Bosma, H.J. Beckers, R.W. Ponds et al. Change in sensory functioning predicts change in cognitive functioning: results from a 6-year follow-up in the Maastricht aging study. J. Am. Geriatr. Soc. 53 (2005) 374-380. doi: 10.1111/j.1532-5415.2005.53152.x. 
66. MH. Sonnet, B. Montaut-Verient, JY. Niemier, M. Hoen, L. Ribeyre, C. Parietti-Winkler. Cognitive abilities and quality of life after cochlear implantation in the elderly. Otol Neurotol. 38(8) (2017) e296-e301. doi: 10.1097/MA0.0000000000001503.

67. C. Völter, L. Götze, S. Dazert, M. Falkenstein, J. P. Thomas. Can cochlear implantation improve neurocognition in the aging population? Clin Interv Aging. 13 (2018) 701712. doi: $10.2147 / \mathrm{CIA} . S 160517$.

68. M. K. Cosetti, J. B. Pinkston, J. M. Flores, D.R. Friedmann, C. B. Jones, J. T. Roland et al. Neurocognitive testing and cochlear implantation: insights into performance in older adults. Clin Interv Aging. 11 (2016) 603-613. doi: 10.2147/CIA.S100255.

69. JA. Deal, MS. Albert, M. Arnold, SI. Bangdiwala, T. Chisolm, S. Davis et al. A randomized feasibility pilot trial of hearing treatment for reducing cognitive decline: Results from the Aging and Cognitive Health Evaluation in Elders Pilot Study. Alzheimers Dement (N Y). 3(3) (2017) 410-415. doi: 10.1016/j.trci.2017.06.003.

70. JA. Deal, AM. Goman, MS. Albert, ML. Arnold, S. Burgard, T. Chisolm et al. Hearing treatment for reducing cognitive decline: design and methods of the Aging and Cognitive Health Evaluation in Elders randomized controlled trial. Alzheimer's \& Dementia 4 (2018) 499-450. https://doi.org/10.1016/j.trci.2018.08.007.

71. GA. Gates. Central presbycusis: an emerging view. Otolaryngology-head and neck surgery. 147(1) (2012) 1-2. https://doi.org/10.1177/0194599812446282.

72. N. Quaranta, F. Coppola, M. Casulli, MR. Barulli, F. Panza, R. Tortelli et al. The prevalence of peripheral and central hearing impairment and its relation to cognition in older adults. Audiol Neurootol. 19(1) (2014) 10-14. doi: 10.1159/000371597.

73. DG. Loughrey, ME. Kelly, GA. Kelley, S Brennan, BA Lawlor. Association of age-related hearing loss with cognitive function, cognitive impairment, and dementia: a systematic review and meta-analysis. JAMA Otolaryngology-- Head \& Neck Surgery. 144(2) (2018) 115-126. doi: 10.1001/jamaoto.2017.2513.

74. DMP. Jayakody, PL. Friedland, RN. Martins, HR. Sohrabi. Impact of aging on the auditory system and related cognitive functions: a narrative review. Front Neurosci. 12 (2018) 125. doi: 10.3389/fnins.2018.00125. 